\title{
Nickelallergie: ohne Patch-Tests keine verlässlichen Zahlen
}

Epidemiologische Studien stützen sich bisweilen auf Eigenangaben. Diese sind praktisch, kostengünstig und erleichtern die Erhebung; ihr Aussagewert muss jedoch hinterfragt werden. Dermatologen der Karolinska-Universität in Stockholm untersuchten die Validität von Patienten-Angaben bezüglich einer Nickel-Allergie.

nsgesamt 369 Frauen aus der Allgemeinbevölkerung zwischen 30 und 40 Jahren nahmen an der Studie teil; die Daten von 355 konnten ausgewertet werden. Sie wurden gebeten, in einem Fragebogen zu verschiedenen Ekzem-relevanten Aspekten wie bestehendes Handekzem, anamnestisch bestehende Atopie, Feuchtarbeit etc. Auskunft zu geben. Die Fragen, die sich auf eine mögliche Nickel-Allergie bezogen, lauteten:

a) Sind Sie sensibilisiert/hypersensitiv/allergisch gegenüber Nickel? und b) Bekommen Sie einen Hautausschlag durch Metallknöpfe, Schmuck oder andere metallene Gegenstände? Danach schlossen sich eine klinische Untersuchung und Epikutantestung als Goldstandard an. Als positiver Befund wurden starke Reaktionen auf Nickelsulfat mit Erythem, Infiltration und Papeln definiert.

Im Epikutantest ermittelte man 30\% Nickel-positive Individuen. Das Vorliegen einer Nickel-Sensibilisierung hatten demgegenüber 40\% der Probandinnen bejaht, Hautreaktionen mit Ausschlägen auf Metallkontakt 35\%.

Man fand, dass der positive prädiktive Wert der beiden Fragen $59 \%$ resp. $60 \%$ war, dass also nur 59 bzw. 60\% derjenigen Frauen, die glaubten, eine Nickel-Allergie zu haben, tatsächlich sensibilisiert waren. Falsch-positive Angaben kamen häufiger bei Frauen vor, die als Kind an einem atopischen Ekzem gelitten hatten. - Möglicherweise, so die Autoren, reagiert atopische Haut leichter gereizt auf Metallkontakt. Ein hohes Maß an Feuchtarbeit oder das Vorliegen eines Handekzems schienen die Aussagen dagegen nicht zu beeinflussen.

Nur wenige der Nickel-positiven Frauen litten unter einer schweren Dermatitis (vier der 108 positiv getesteten), bei den meisten waren die Reaktionen mild, und 24\% wussten gar nichts von der Sensibilisierung.

Fazit: Nicht nur, um die Auswirkungen der EU-NickelDirektive zu prüfen, ist es interessant, die Entwicklung dieser Kontaktallergie zu verfolgen. Die beiden verwendeten Fragen erwiesen sich aber als nicht besonders brauchbar für die Ermittlung der Prävalenz; das Ergebnis sind tendenziell zu hohe Raten.

wpa 